\title{
Rational design of artificial riboswitches based on ligand-dependent modulation of internal ribosome entry in wheat germ extract and their applications as label-free biosensors
}

\author{
ATSUSHI OGAWA \\ Senior Research Fellow Center, Ehime University, Matsuyama, Ehime 790-8577, Japan
}

\begin{abstract}
Riboswitches are RNA elements in mRNA that control gene expression in cis in response to their specific ligands. Because artificial riboswitches make it possible to regulate any gene with an arbitrary molecule, they are expected to function as biosensors, in which the output is easily detectable protein expression. I report herein a fully rational design strategy for artificially constructing novel riboswitches that work in a eukaryotic cell-free translation system (wheat germ extract). In these riboswitches, translation mediated by an internal ribosome entry site (IRES) is promoted only in the presence of a specific ligand (ON), while it is inhibited in the absence of the ligand (OFF). The first rationally designed riboswitch, which is regulated by theophylline, showed a high switching efficiency and dependency on theophylline. In addition, based on the design of the theophylline-dependent riboswitch, other three kinds of riboswitches controlled by FMN, tetracycline, and sulforhodamine B, were constructed only by calculating the $\Delta G$ value of one stem-loop structure. The rational design strategy described herein is therefore useful for easily producing various ligand-dependent riboswitches, which are available as biosensors for detecting their ligands.
\end{abstract}

Keywords: biosensor; riboswitch; IRES; RNA aptamer

\section{INTRODUCTION}

Riboswitches are ligand-dependent gene regulators that have been found in untranslated regions (UTR) of mRNA, mainly in prokaryotes and rarely in eukaryotes (Roth and Breaker 2009). They are composed of two domains, an aptamer and an expression platform, and control the expression of a downstream (or upstream in some cases) gene in cis without the need for any proteins. They carry out this function by changing their conformations upon binding to their specific ligands in the aptamer domain to promote/inhibit transcription termination, translation initiation, mRNA degradation, or splicing in the expression platform (Roth and Breaker 2009). While the ligands are principally metabolites relevant to the regulated gene in natural riboswitches, artificial riboswitches make it possible to regulate any gene with an arbitrary

Reprint requests to: Atsushi Ogawa, Senior Research Fellow Center, Ehime University, 3 Bunkyo-cho, Matsuyama, Ehime 790-8577, Japan; e-mail: a-ogawa@ccr.ehime-u.ac.jp; fax: 81899278450.

Article published online ahead of print. Article and publication date are at http://www.rnajournal.org/cgi/doi/10.1261/rna.2433111. molecule. They are therefore expected to work as various molecule-dependent gene regulators in vivo (Suess and Weigand 2008; Topp and Gallivan 2010) as well as in vitro biosensors for detecting various molecules (Ogawa 2009 and references therein).

In terms of the latter applications, by using a posttranscriptional up-regulation-type riboswitch (pt-ONriboswitch) and a reporter gene (e.g., luciferase) as the regulated gene in an appropriate cell-free translation system, label-free biosensors can easily be constructed because the riboswitch ligand (i.e., analyte) up-regulates the expression of the reporter gene depending on its concentration. However, whereas many in vivo applications of artificial riboswitches in both prokaryotes and eukaryotes have been reported thus far (Werstuck and Green 1998; Grate and Wilson 2001; Harvey et al. 2002; Suess et al. 2003, 2004; Desai and Gallivan 2004; Kim et al. 2005; Nomura and Yokobayashi 2007; Weigand and Suess 2007; Win and Smolke 2007; Ogawa and Maeda 2008a; Wieland and Hartig 2008; Weigand et al. 2008), there have been few examples of their applications as in vitro biosensors. I and Maeda have previously reported two types of prokaryotic riboswitch-based in vitro biosensors that were 
rationally designed using aptazymes, which are functional RNA and cleave themselves in response to their specific ligand molecules (Ogawa and Maeda 2007, 2008b). Most recently, I have reported a eukaryotic riboswitch-based in vitro biosensor that was also rationally constructed by the use of the aptazyme (Ogawa 2009). Yokobayashi et al. have reported that in vivo-selected prokaryotic riboswitches are available as in vitro biosensors (Muranaka et al. 2009).

Although the rational design or selection strategy for these riboswitches that function in vitro could be used for constructing a wide variety of in vitro biosensors for arbitrary analytes, there are some issues to be resolved. In the selection strategy, basically, each riboswitch corresponding to a target analyte must be selected in vivo one by one. In the rational design strategy of aptazyme-based riboswitches, while it is easy to construct various riboswitches using various aptazymes, each aptazyme in response to a target analyte must be selected via in vitro selection, in most cases using independently selected aptamers (Breaker 2002). In addition, the aptazyme activity (in limited sense, ribozyme activity) is sensitive to magnesium concentrations in a narrow range and thus is quite different between in vitro and in vivo: the prokaryotic aptazyme-based riboswitch optimized in vitro relatively drops its switching efficiency in vivo (Ogawa and Maeda 2008a); in contrast, another prokaryotic aptazymebased riboswitch optimized in vivo shows much lower switching efficiency in vitro due to the too fast cleavage in the absence of its ligand (Wieland and Hartig 2008). On the other hand, it has been reported that prokaryotic ribozymefree aptamer-based pt-ON-riboswitches selected in vivo function in vitro with comparable characteristics without optimizing magnesium concentrations (Muranaka et al. 2009). Therefore, given the future applicability in vivo or compatibility between in vitro and in vivo, a rational design strategy for engineering ribozyme-free aptamer-based ptON-riboswitches that do not involve mRNA cleavage is expected to be established, partially because a number of methods for easily selecting aptamers have been reported and many aptamers have already been selected in contrast to aptazymes (Wilson and Szostak 1999).

Nonetheless, to the best of my knowledge, no generalized rational design method for constructing ribozyme-free aptamer-based pt-ON-riboswitches has been reported either in vitro or in vivo, although there have been reported a rational design method of ribozyme- and aptamer-based pt-ONriboswitches (Win and Smolke 2007, 2008). I therefore have decided to establish a method for rationally constructing ptON-riboswitches in vitro by the use of aptamers without ribozymes. Moreover, I chose to design eukaryotic ribozymefree aptamer-based pt-ON-riboswitches because there has been no example of those obtained, even via selection methods, in contrast to prokaryotic ribozyme-free aptamer-based pt-ON-riboswitches selected in vivo (Desai and Gallivan 2004; Nomura and Yokobayashi 2007; Muranaka et al. 2009) or eukaryotic ribozyme-free aptamer-based pt-OFF-riboswitches (down-regulation-type) (Werstuck and Green 1998; Grate and Wilson 2001; Harvey et al. 2002; Suess et al. 2003; Kim et al. 2005; Weigand and Suess 2007; Weigand et al. 2008).

It is, however, challenging to design ribozyme-free aptamer-based pt-ON-riboswitches functioning in a eukaryotic translation system, unlike in a prokaryotic translation system. The principal reason for the difficulty is that the translation mode differs between eukaryotes and prokaryotes: the ribosome loads on a $5^{\prime}$ terminus of mRNA in the former, but on the ribosome-binding site (RBS, i.e., Shine-Dalgarno sequence) in mRNA in the latter (Ogawa 2009). In the typical prokaryotic ribozyme-free aptamer-based pt-ONriboswitches selected in vivo, an aptamer and an anti-RBS sequence, which is in most cases a part of the aptamer domain, reside upstream from the RBS (Desai and Gallivan 2004; Nomura and Yokobayashi 2007). In these riboswitches, while the anti-RBS sequesters the RBS from the ribosome, inhibiting translation, the aptamer-ligand complex formation makes the anti-RBS release from the RBS, promoting translation. On the other hand, in a eukaryotic translation system, it is impossible to insert the aptamer before a $5^{\prime}$ cap. If the aptamer is inserted in the $5^{\prime}$ UTR after the $5^{\prime}$ cap, the aptamer-ligand complex prevents the ribosome from scanning the mRNA, so that the eukaryotic $5^{\prime}$-terminus-mediated translation mode allows us to construct only pt-OFF-riboswitches with aptamers (without ribozymes).

I therefore focused on another translation mode working in eukaryotes: translation from an internal ribosome entry site (IRES) (Hellen and Sarnow 2001). The eukaryotic ribosome can bind to the IRES and start translation as well as when the prokaryotic ribosome binds to the RBS. The IRES is thus expected to be used as an expression platform in constructing eukaryotic ribozyme-free aptamer-based riboswitches like the RBS in prokaryotic riboswitches. I report herein the fully rational design of novel eukaryotic ptON-riboswitches based on ligand-dependent modulation of IRES-mediated translation in wheat germ extract (Madin et al. 2000) and their applications as in vitro biosensors.

\section{RESULTS}

\section{Optimization of an IRES-mediated translation system}

First, I optimized mRNA toward efficient IRES-mediated translation by using the Plautia stali intestine virus (PSIV) IRES in the intergenic region of the PSIV genome as the IRES (Fig. 1A; Sasaki and Nakashima 1999) and firefly luciferase (F-Luc) as a reporter protein translated via the IRES. The PSIV IRES was chosen because it has been well studied (Sasaki and Nakashima 1999, 2000; Kanamori and Nakashima 2001; Shibuya et al. 2003, 2004), its 3D structure has been resolved by X-ray (Pfingsten et al. 2006), and moreover it functions well in many types of eukaryotic translation systems: not only in insect cells, but also in rabbit reticulocyte lysate (Kanamori and Nakashima 2001) and in 
A

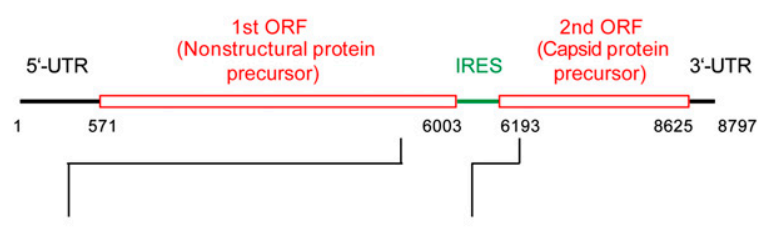

B
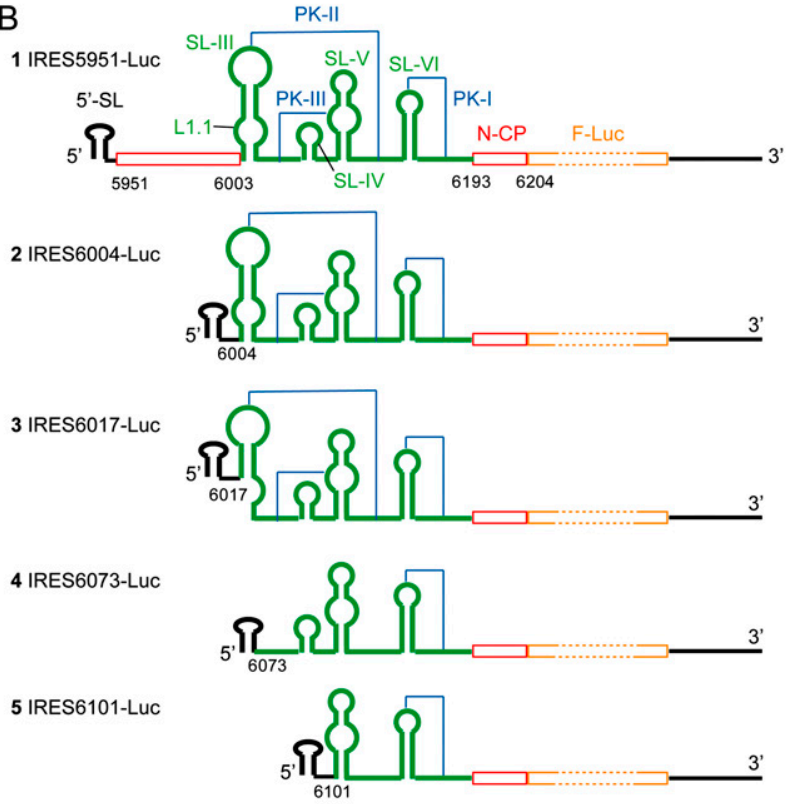

C

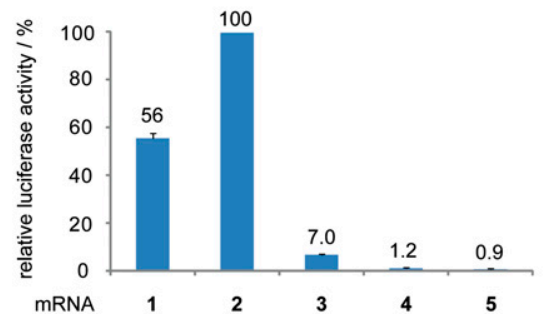

FIGURE 1. Optimization of IRES-mediated translation. (A) Schematic diagram of the PSIV genome. The numbers represent nucleotide positions. $(B)$ Five variants of mRNA coding luciferase (F-Luc) with various $5^{\prime}$-terminal lengths of PSIV IRES in the $5^{\prime}$ UTR. The numbers under the genes correspond to nucleotide positions in the PSIV genome. The tertiary structure of the IRES is based on previous reports (Kanamori and Nakashima 2001; Shibuya et al. 2003; Pfingsten et al. 2006). Green and cyan letters indicate important structures and interactions for efficient IRES-mediated translation: (L) loop; (SL) stem-loop; (PK) pseudoknot. The cyan lines represent pseudoknots. 5'-SL and N-CP indicate a $5^{\prime}$-terminal stem-loop structure and four $\mathrm{N}$-terminal amino acids of the capsid protein precursor, respectively. See also Figure $2 \mathrm{~A}$ for the whole IRES sequence. $(C)$ The relative activities of luciferase translated from various mRNAs compared to that from mRNA 2. All data are averages with standard deviations of three independent experiments. Only averages are shown above each graph.

wheat germ extract (Shibuya et al. 2003). Although the region of PSIV IRES required for efficient IRES-mediated translation has been examined elsewhere, there have been somewhat different results regarding the $5^{\prime}$ boundary due to the effect of the upstream open reading frame (ORF) (Sasaki and Nakashima 1999; Kanamori and Nakashima 2001). Thus, five variants of mRNA with various $5^{\prime}$-terminal lengths of the IRES fused to the F-Luc coding sequence were prepared to reexamine the $5^{\prime}$ boundary (Fig. 1B, 1-5). In terms of the $3^{\prime}$ boundary, 12 bases corresponding to four $\mathrm{N}$-terminal amino acids of the second ORF (coding capsid protein precursor; $\mathrm{N}-\mathrm{CP}$ ) were inserted between the IRES and the F-Luc coding sequence in all variants in order to enhance IRES-mediated translation (Sasaki and Nakashima 1999). In addition, to inhibit $5^{\prime}$-terminus-mediated translation, a stem-loop structure $\left(5^{\prime}-\mathrm{SL}\right)$ was added to the $5^{\prime}$ terminus of
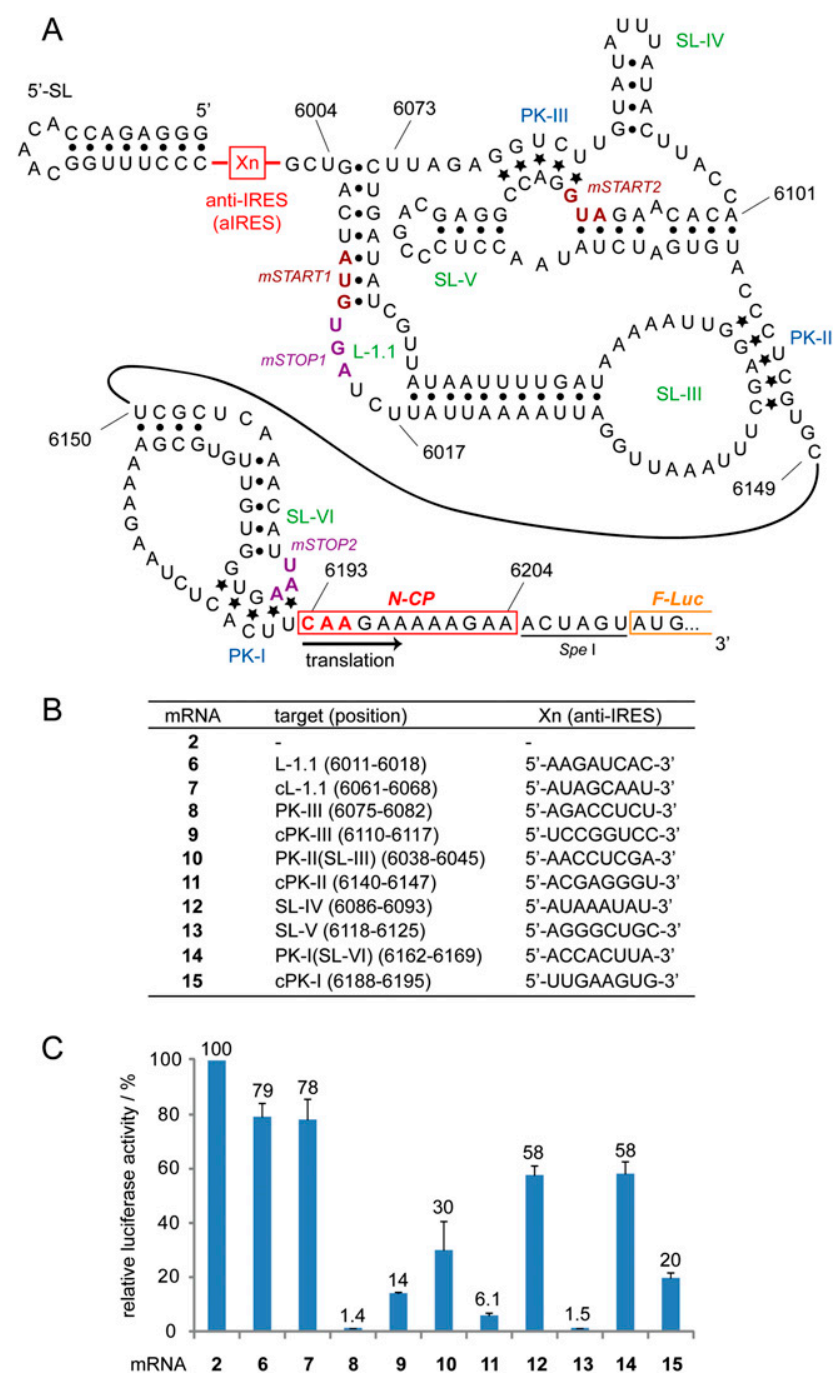

FIGURE 2. Inhibition of IRES-mediated translation by the aIRES. (A) The sequence and tertiary structure of PSIV IRES. The numbers represent nucleotide positions in the PSIV genome. Asterisks and dots represent base-pair interactions for pseudoknots and helical duplexes, respectively. mSTART and $\mathrm{mSTOP}$ indicate the start and stop codons for mimic genes, respectively. Various aIRES were inserted into Xn. $(B)$ The sequences of various aIRES and their target in the PSIV IRES. (C) The relative activities of luciferase translated from various mRNAs compared to that from mRNA 2. All data are averages with standard deviations of three independent experiments. Only averages are shown above each graph. 
these mRNAs. As reported previously, 5' -terminus-mediated translation can be inhibited by the $5^{\prime}$-terminal duplex of mRNA (Babendure et al. 2006; Ogawa 2009) and mimic genes in the 5' UTR (Ogawa 2009). Fortunately, the PSIV IRES has two mimic genes: two AUG codons and the corresponding ORFs (Fig. 2A, mSTART1-mSTOP1 and mSTART2-mSTOP2), one of which includes the Kozak sequence for effective translation initiation (mSTART2) (Kozak 1989). Therefore, these mimic genes, not the genuine gene (coding F-Luc), are expected to be dominantly translated, even if the ribosome loads on the $5^{\prime}$ terminus.

Figure 1C shows the activities of luciferase translated from five variants (1-5) in wheat germ extract (Madin et al. 2000). The variant showing the highest activity is mRNA 2, the translation efficiency of which is $\sim 40 \%$ compared to that of $5^{\prime}$-terminus-mediated translation of mRNA with the most effective enhancer sequence in the 5' UTR (Kamura et al. 2005; Ogawa 2009). This variant (2) has the full length of the intergenic region as the IRES, suggesting that the $5^{\prime}$ boundary of PSIV IRES is around 6004, which is in accordance with one of previous reports (Kanamori and Nakashima 2001). The longer IRES (1) is rather less effective, probably because it includes a part of the first ORF adversely affecting the IRES conformations. On the other hand, the shorter IRES (4 and 5) exhibited almost no translation ability due to a lack of several domains necessary for an active IRES structure. These low translation efficiencies of $\mathbf{4}$ and $\mathbf{5}$ indicate that $5^{\prime}$-terminus-mediated translation of the genuine gene was almost totally inhibited in all five variants.

\section{Inhibition of IRES-mediated translation by an anti-IRES sequence}

In constructing ON-switches, one of the most significant goals is to suppress translation in the absence of the ligand. To achieve this objective, I searched for anti-IRES sequences (aIRES) to inhibit IRES-mediated translation from several candidates, which were inserted between the $5^{\prime}$-SL and the IRES (Fig. 2). The candidates were 8-mer complementary sequences to 10 parts of IRES that are considered to play important roles in forming the precise conformations of the IRES (three pseudoknots: PK-I; PK-II; PK-III) or in binding to the ribosome (two stem-loops for binding to the 40S ribosome: SL-IV and SL-V; one loop for binding to the $60 \mathrm{~S}$ ribosome; L-1.1) (Fig. 2B; Kanamori and Nakashima 2001; Pfingsten et al. 2006). Of these candidates, two aIRES complementary to a $5^{\prime}$ side of PK-III (in 8) and SL-IV (in 13) showed high inhibition effects (Fig. 2C) comparable to the low translation efficiency of mRNA 4 lacking several important domains. These results, indicating that these aIRES form a duplex with their target to inhibit IRES function (see Fig. 3B for the case of anti-PKIII), were as expected. This is because the position of aIRES insertion is sterically close to the $5^{\prime}$ side of PK-III and SLIV in the anti-parallel direction according to the X-ray IRES structure (Pfingsten et al. 2006), thus allowing for easy duplex formation, while other candidates are far from targets and/or are in the parallel direction, other than one exception (anti-L-1.1). Although the anti-L-1.1 (in 6) is adjacent to the target in the anti-parallel direction to form a stem-loop, it showed no inhibition effect, despite the low translation efficiency of mRNA 3 lacking its target, the $5^{\prime}$ side of L-1.1. This result was probably due to the $40 \mathrm{~S}$
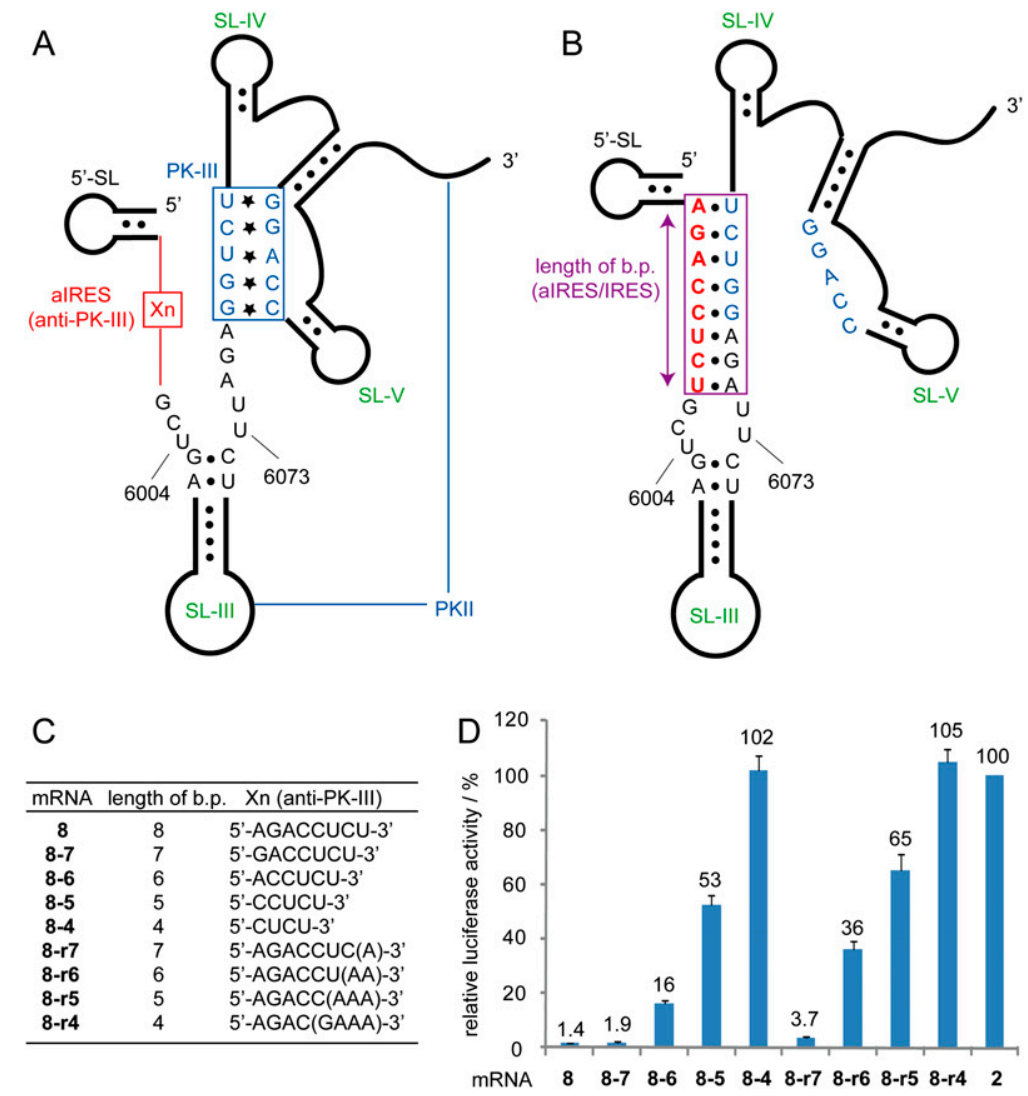

FIGURE 3. The effects of the anti-PK-III length on translation inhibition. $(A, B)$ Schematic diagram of a $5^{\prime}$ terminus of mRNA. An active IRES structure with PK-III $(A)$ and an inactive IRES structure with an anti-PK-III/5'-side-PK-III (aIRES/IRES) duplex $(B)$. $(C)$ The sequences of various anti-PK-III (aIRES) for various lengths of a duplex with the $5^{\prime}$ side of PK-III (length in base pairs). When the $3^{\prime}$ terminus was shortened (8-r7-8-r4), extra bases (in parentheses) derived from the original genome sequence were added to the $3^{\prime}$ terminus to adjust the duplex position. $(D)$ The relative activities of luciferase translated from various mRNAs compared to that from mRNA 2. All data are averages with standard deviations of three independent experiments. Only averages are shown above each graph. 
ribosome binding to the IRES before the $60 \mathrm{~S}$ ribosome, with interactions between the $40 \mathrm{~S}$ and the IRES inducing dehybridization of the duplex (anti-L-1.1/L-1.1). This hypothesis is consistent with a previous report showing that L-1.1 is significant to $60 \mathrm{~S}$ binding, but not to $40 \mathrm{~S}$ binding (Pfingsten et al. 2006).

Next, to examine the effects of the aIRES length on translation, I varied the lengths of anti-PK-III in mRNA 8, which showed a slightly higher inhibition of IRES-mediated translation than another promising candidate, mRNA 13 (Fig. 3). In both cases, when the $5^{\prime}$ terminus and $3^{\prime}$ terminus were shortened, 4-mers (8-4, 8-r4) showed no translation inhibition due to the lack of duplex formation with the 5 ' side of PK-III, while 7 8-mer was found to be required for full inhibition of IRES-mediated translation.

\section{Restoration of IRES-mediated translation by an anti-anti-IRES sequence}

In ON-riboswitches, inhibited translation in the absence of the ligand must be restored by conformational changes induced by the ligand. In artificial prokaryotic ribozyme-free aptamer-based pt-ON-riboswitches obtained by selection methods, an aptamer domain plays a dual role as both an aptamer and an anti-RBS, so that the aptamer-ligand complex formation directly induces release of the anti-RBS from the RBS to restore translation (Desai and Gallivan 2004; Nomura and Yokobayashi 2007). However, it is difficult to obtain aptamers including the same sequence as the aIRES identified above, partially because the required length of the aIRES for translation inhibition is longer than that of the anti-RBS. Moreover, if it is possible to isolate such a riboswitch, it is also hard to apply the isolated riboswitch to the design of other riboswitches. To rationally construct IRES-based pt-ON-riboswitches, they must include a part that can restore IRES-mediated translation in addition to an aptamer and an aIRES. The candidates are, needless to say, anti-sequences to the aIRES (i.e., anti-anti-IRES; aaIRES). Thus, several aaIRES sequences with various lengths complementary to the anti-PKIII (aIRES in 8) were inserted before the aIRES, with five bases (loop) intervening between them to form a stem-loop structure, so as to determine if they could restore IRES-mediated translation (Fig. 4). As a result, while the shorter aaIRES (3-6-mer, aa3-aa6) showed almost no effect, the same length (8-mer, aa8) of aaIRES as the aIRES was found to form a duplex with the aIRES to restore IRESmediated translation, wherein the rate of recovery was $\sim 80 \%$ compared to mRNA
2 (Fig. 4C). This result suggests that modulation of duplex formation between the aaIRES and the aIRES should lead to that of PK-III formation in the IRES, and thus to that of IRES-mediated translation. Incidentally, the longer aaIRES (9- and 10-mer, aa9 and aa10, respectively) restored IRESmediated translation less effectively than aa8. This is probably because the extra one or two bases adversely affected the IRES structure.

\section{Design strategy for a ligand-inducible IRES-mediated translation system}

The strategy for designing a ligand-inducible IRES-mediated translation system is as follows (see also Fig. 5): an aptamer sequence that can tightly and specifically bind to its ligand is inserted between the aaIRES and the aIRES in aa8 instead of the intervening loop; a modulator sequence (MS) that is complementary to a sequence composed of a $3^{\prime}$ end of the aaIRES and a $5^{\prime}$ end of the aptamer is inserted before the aaIRES (after the $5^{\prime}$-SL) to modulate the aaIRES/ aIRES duplex formation depending on the absence (OFF) or presence $(\mathrm{ON})$ of the ligand; the length of the MS is optimized so that the ratio of translation efficiency in the ON state to that in the OFF state (ON/OFF ratio) is higher. In this strategy, the MS is expected to inhibit the aaIRES/ aIRES duplex formation by forming a stem-loop (MS-SL, dotted square in Fig. 5) to suppress IRES-mediated translation in the OFF state (Fig. 5, left). In contrast, in the ON state, it is expected to be released from the aptamer and the aaIRES by the aptamer-ligand binding to promote the aaIRES/aIRES duplex formation and thus enhance IRESmediated translation (Fig. 5, right).
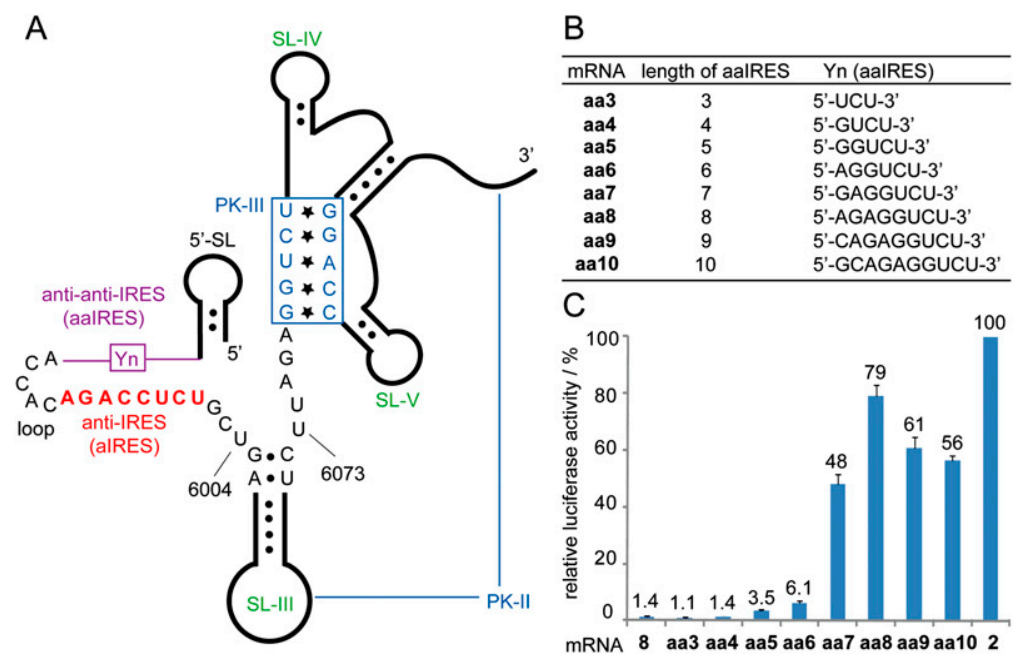

FIGURE 4. Translation restoration by the aaIRES. (A) Schematic diagram of a $5^{\prime}$ terminus of mRNA: an active IRES structure with PK-III and an aaIRES/aIRES duplex. Various lengths of aaIRES were inserted into Yn. $(B)$ The sequences of various lengths of aaIRES. $(C)$ The relative activities of luciferase translated from various mRNAs compared to that from mRNA 2. All data are averages with standard deviations of three independent experiments. Only averages are shown above each graph. 


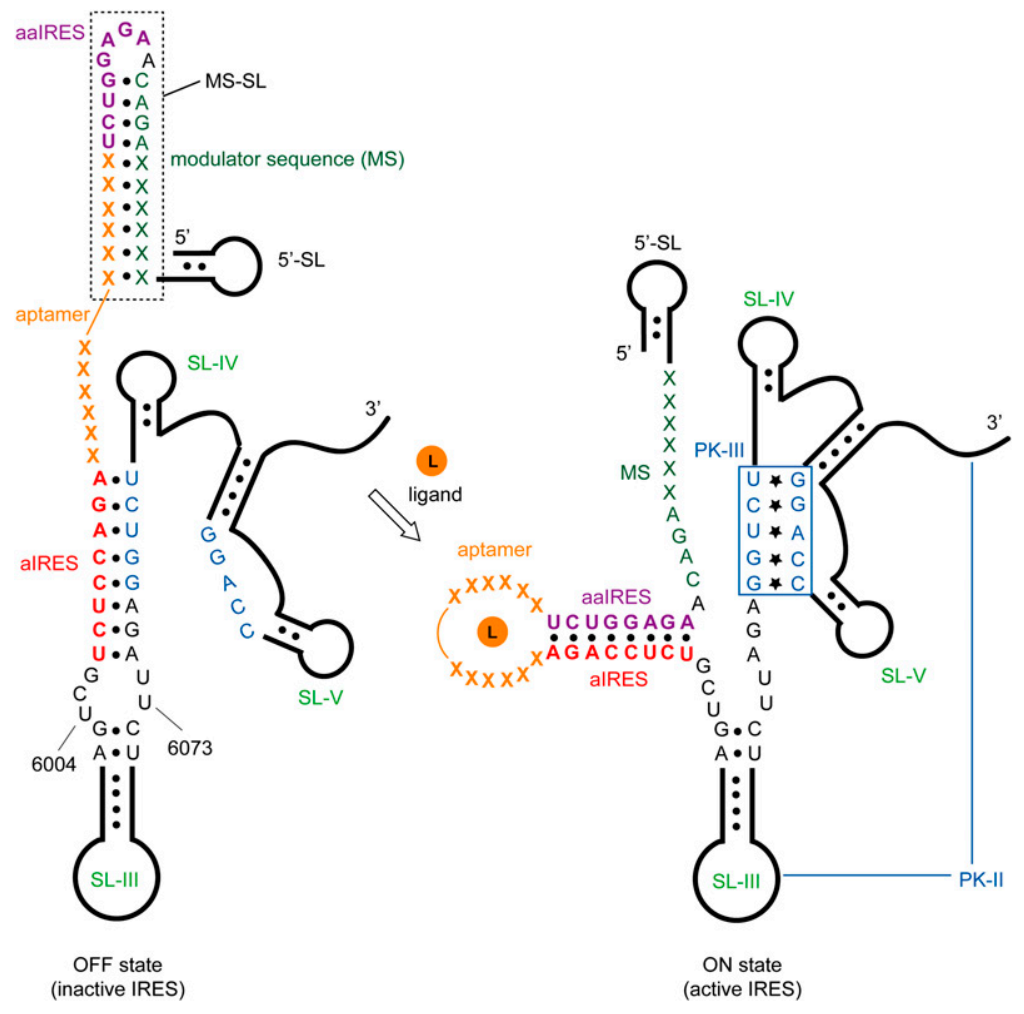

FIGURE 5. Schematic diagram of a $5^{\prime}$ terminus of mRNA in the ligand-inducible IRESmediated translation system. In the absence of a ligand, a modulator sequence (MS, thick green) hybridizes to a $3^{\prime}$ part of an aaIRES (purple) and a $5^{\prime}$ part of an aptamer for the ligand (orange) to form a stem-loop structure (MS-SL, dotted square), inducing aIRES/IRES duplex formation and thus inhibiting IRES-mediated translation (left, inactive IRES structure, OFF state). One base $(A)$ was inserted between the MS and the aaIRES for a stable loop structure. In the presence of the ligand (orange circle), the ligand-aptamer complex releases the MS from the aptamer and the aaIRES to induce aaIRES/aIRES duplex and PK-III formation, thus promoting IRES-mediated translation (right, active IRES structure, ON state). ratio of IRES-mediated translation efficiency (Fig. 6). As a result, the 5-mer of this part of MS (totally 9-mer of MS, theo5) showed the highest ON/OFF ratio (Fig. 6C). While the shorter MS did not adequately inhibit aaIRES/aIRES duplex formation in the OFF state, the longer MS than 9-mer almost completely sequestered the aaIRES from the aIRES, resulting in translation efficiencies as low as mRNA 8. In contrast, the longer MS bound tightly to the aptamer, showing a lower translation efficiency in the $\mathrm{ON}$ state. The most efficient mRNA, theo5, showed high theophylline dependency and sensitivity (detection limit $\sim 3 \mu \mathrm{M}$ ) (Fig. 6D), which indicates that this IRESbased riboswitch is available as a biosensor. theo5 also showed high specificity: 1 $\mathrm{mM}$ caffeine, which has almost the same structure as theophylline except for the N7 methyl group, has no effect on the translation of theo5 (Fig. 6E, left). In addition, a mutant of theo5 (m-theo5), which has double mutations in nucleotides involved in theophylline binding (C22A and U24A), showed no response to $1 \mathrm{mM}$ theophylline (Fig. 6E, right). These results clearly indicate that the specific binding between theophylline and the aptamer domain in theo5 switches IRES-mediated translation on.

\section{Rational design of a theophylline-inducible IRES-mediated translation system and its application as a biosensor}

A first design of the ligand-inducible IRES-mediated translation system was achieved by using a theophylline aptamer, which was obtained by an in vitro selection method (Jenison et al. 1994) and has been used for constructing many artificial riboswitches (Harvey et al. 2002; Suess et al. 2003; Desai and Gallivan 2004; Kim et al. 2005; Ogawa and Maeda 2007, 2008a,b; Win and Smolke 2007; Ogawa 2009) because of its high binding and discriminating ability for its ligand, theophylline. The length of a $3^{\prime}$ part of MS hybridizing to the aaIRES was fixed to 4-mer ( $5^{\prime}$-AGAC- $\left.3^{\prime}\right)$. This is because 4-mer of aIRES showed no translation inhibition, as described above (Fig. 3, 8-r4), although a complementary sequence to the aaIRES is the same as the aIRES, which is highly likely to bind to the IRES to inhibit IRES-mediated translation even if the aaIRES/aIRES duplex forms only in the ON state. The length of the $5^{\prime}$ part hybridizing to the theophylline aptamer was optimized toward a high ON/OFF

\section{Rational design of other-ligand-inducible IRES-mediated translation systems}

To apply the design of theophylline-dependent riboswitches to those of other-ligand-dependent systems, the free energies $(\Delta G)$ of MS-SL in theophylline-dependent riboswitches were calculated by RNAstructure (Fig. 6C; Mathews et al. 2004). As a result of the calculation, $\Delta G$ of less than $\sim-11.7 \mathrm{kcal} / \mathrm{mol}$ was found to be required for the inhibition of aaIRES/aIRES duplex formation. A stronger MS-SL such as in theo8 is, nonetheless, difficult to dehybridize in the ON state. I therefore constructed other-ligand-inducible riboswitches with MS-SL having $\Delta G$ of $\sim-11.7 \mathrm{kcal} / \mathrm{mol}$ by inserting in vitro-selected aptamers for FMN (Burgstaller and Famulok 1994), tetracycline (Berens et al. 2001), and sulforhodamine B (Holeman et al. 1998) between the aaIRES and the aIRES instead of the theophylline aptamer (Fig. 7A,B). Figure 7C shows the translation efficiencies of these riboswitches in the absence or presence of their ligands $(300 \mu \mathrm{M})$. In all three variants (FMN4, tc7, sr4), the translation efficiency in the 


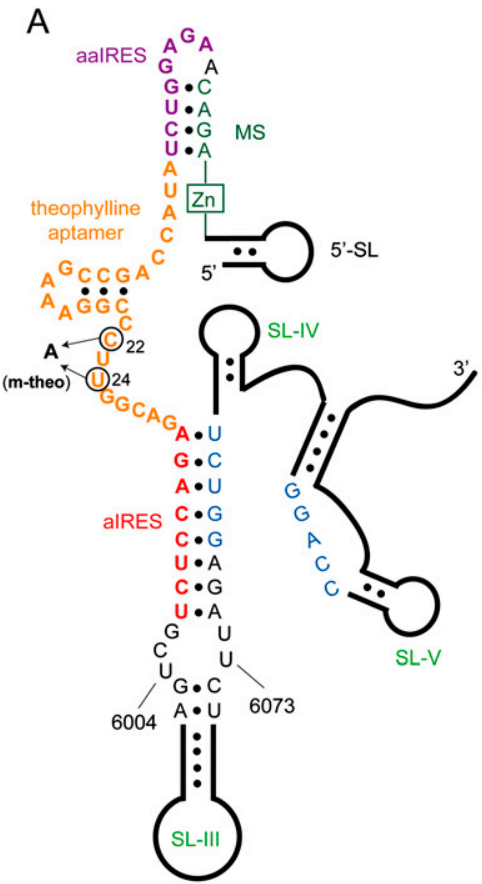

$\mathrm{D}$

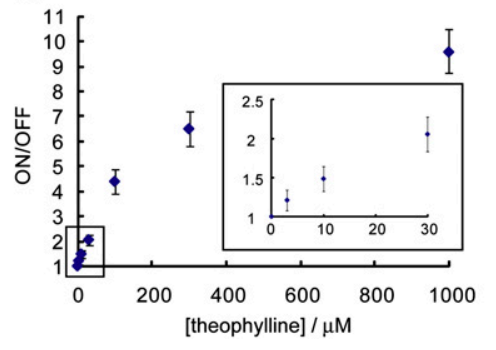

B

\begin{tabular}{lcl}
\hline mRNA & length of MS & \multicolumn{1}{c}{ MS (Zn-AGAC) } \\
\hline theo2 & 6 & 5'-AU-AGAC-3' \\
theo3 & 7 & 5'-UAU-AGAC-3' \\
theo4 & 8 & 5'-GUAU-AGAC-3' \\
theo5 & 9 & 5'-GGUAU-AGAC-3' \\
theo6 & 10 & 5'-UGGUAU-AGAC-3' \\
theo7 & 11 & 5'-CUGGUAU-AGAC-3' \\
theo8 & 12 & 5'-GCUGGUAU-AGAC-3' \\
\hline
\end{tabular}
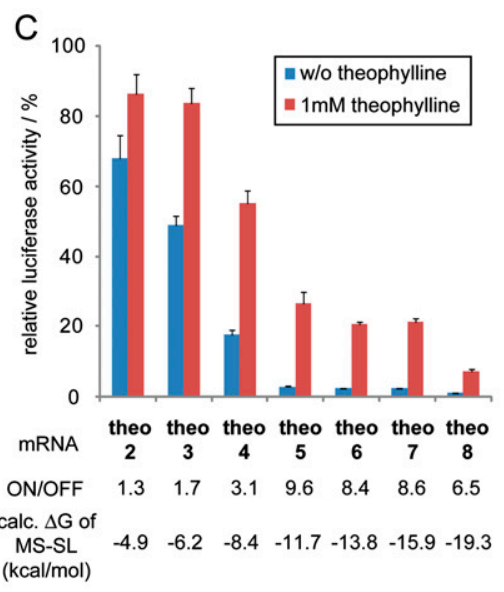

$\mathrm{E}$

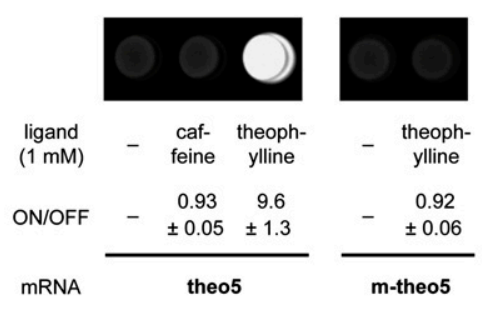

FIGURE 6. Rational design of a theophylline-dependent IRES-mediated translation system. (A) Schematic diagram of a $5^{\prime}$ terminus of mRNA (OFF state, inactive IRES structure). A $3^{\prime}$ part of MS hybridizing to a $3^{\prime}$ end of aaIRES was fixed to $5^{\prime}$-AGAC-3'. Various lengths of the $5^{\prime}$ part of MS were inserted into Zn. Nucleotides in the aptamer domain are numbered according to Zimmermann et al. (1997). (B) The sequences of various MS. (C) The relative activities of luciferase translated from various mRNAs, compared to that from mRNA 2 , in the absence (cyan) or presence of $1 \mathrm{mM}$ theophylline (magenta). All data are averages with standard deviations of three independent experiments. Average ON/OFF efficiencies and calculated $\Delta G$ 's of MS-SL are shown below the graph. (D) Theophylline dependency of theo5. (Inset) Expansion at low concentrations of theophylline. (E) Chemiluminescence images of luciferase translated from theo5 (left) or $\mathbf{m}$-theo5 (right), which has double mutations in the aptamer domain of theo5 (C22A and U24A as shown in $A$ ), in the absence or presence of $1 \mathrm{mM}$ theophylline or caffeine.

absence of their ligand (OFF state) was as low as that of theo5 in the OFF state and increased in the presence of their ligand (ON state), as expected. The tetracycline-dependent riboswitch (tc7) in particular showed the highest switching efficiency $(\mathrm{ON} / \mathrm{OFF}=\sim 30)$, which was 4.5 -fold higher than that of theo5. Given the fact that a reported dissociation constant $\left(K_{\mathrm{D}}\right)$ of theophylline aptamer and tetracycline aptamer is $0.3 \mu \mathrm{M}$ (Jenison et al. 1994) and $<1 \mathrm{nM}$ (Müller et al. 2006), the much higher switching efficiency of tc7 can be attributed to the stronger binding between tetracycline and its aptamer. On the other hand, FMN4 with the FMN aptamer and sr4 with the sulforhodamine B aptamer showed comparable switching efficiencies to theo5. These results are in agreement with the fact that both the FMN aptamer and the sulforhodamine B aptamer have similar values of $K_{\mathrm{D}}$ to the theophylline aptamer (0.5 $\mu \mathrm{M}$ and $0.3 \mu \mathrm{M}$, respectively) (Burgstaller and Famulok 1994; Holeman et al. 1998). Figure 7D shows that no ligands at concentrations of $300 \mu \mathrm{M}$ (up to $1 \mathrm{mM}$ for theophylline) have an effect on IRES-mediated luciferase expression.

\section{DISCUSSION}

The rational design strategy for constructing ligand-inducible IRES-mediated translation systems in wheat germ extract has been established here. The key to the design is the use of five parts fused to a $5^{\prime}$ terminus of the IRES: (1) an aIRES to inhibit IRES function by hybridizing to an important element of the IRES; (2) an aptamer to bind to the ligand; (3) an aaIRES to restore IRES function by hybridizing to the aIRES; (4) an MS to modulate aIRES/aaIRES duplex formation with or without the ligand; and (5) a 5'-SL to inhibit $5^{\prime}$-terminus-mediated translation. The aIRES and the aaIRES that have been selected and optimized here are available without modifications. The aptamer and its ligand are arbitrary unless the aptamer includes a sequence distorting the riboswitch system or the ligand dramatically inhibits translation. The $5^{\prime}-\mathrm{SL}$ is changeable in accordance with a transcription system used. Although the MS may be optimized, all one has to do is calculate $\Delta G$ of MS-SL and choose an appropriate MS. In fact, in the present study, three kinds of IRES-based pt-ONriboswitches regulated by their corresponding ligands were easily constructed only by adjusting their $\Delta G$ of MS-SL to that in the first-designed and optimized theophyllinedependent riboswitch. Therefore, if an aptamer for a certain ligand is obtained, it is easy to produce the corresponding IRES-based riboswitch up-regulating the expression of a downstream gene in response to the ligand.

As described above, these riboswitches are available as biosensors to detect their ligands because the output is protein expression, which is easily detectable. No label is required by using a reporter gene as the regulated gene. In 
A
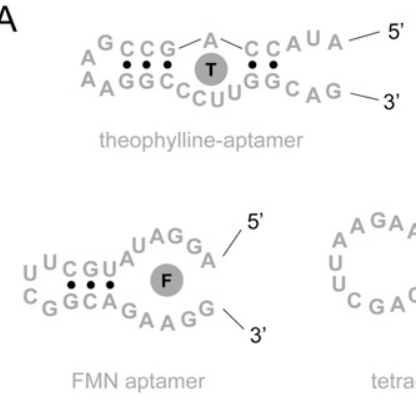

B

\begin{tabular}{lcclc}
\hline mRNA & ligand & length of MS & MS (Zn-AGAC) & $\Delta$ G of MS-SL (kcal/mol) \\
\hline theo5 & theophylline (theo) & 9 & 5'-GGUAU-AGAC-3' & -11.7 \\
FMN4 & FMN & 8 & 5'-UCCU-AGAC-3' & -11.6 \\
tc7 & tetracycline (tc) & 11 & 5'-AUGUUUU-AGAC-3' & -11.9 \\
sr4 & sulforhodamine B (sr) & 8 & 5'-AGCG-AGAC-3' & -12.8 \\
\hline
\end{tabular}
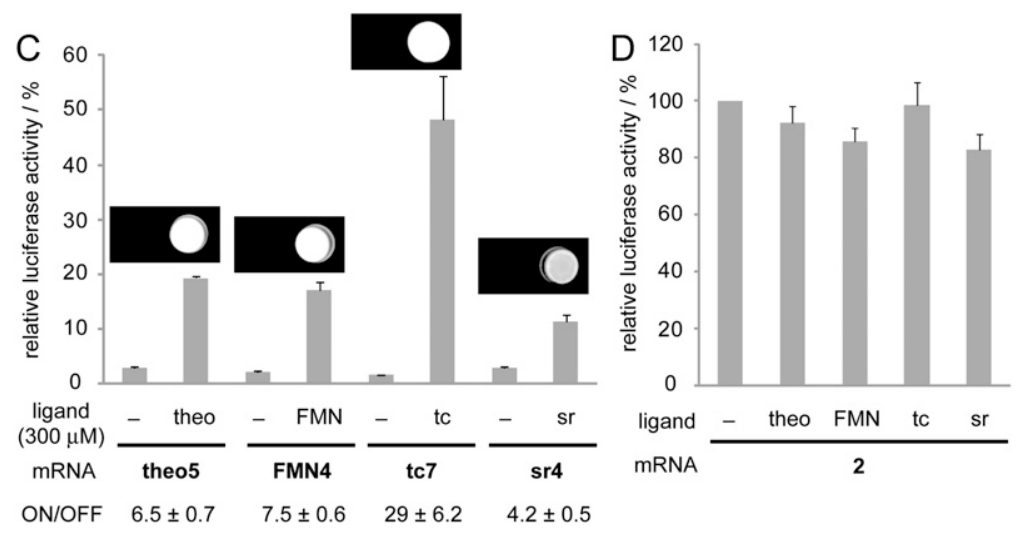

FIGURE 7. Rational design of various ligand-dependent pt-ON-riboswitches. $(A)$ Various aptamer sequences and predicted secondary structures in the presence of their ligands. $(B)$ The sequences of MS and calculated $\Delta G$ of MS-SL in various riboswitches. $(C)$ The relative activities of luciferase translated from various mRNAs in the absence or presence of the ligand $(300 \mu \mathrm{M})$, compared to that from mRNA 2 without any ligands. All data are averages with standard deviations of three independent experiments. Chemiluminescence images and average ON/OFF efficiencies with standard deviations are shown above and below the graph, respectively. $(D)$ Control experiments investigating the effects of various ligands on PSIV IRES-mediated luciferase expression. The concentrations of ligands except theophylline $(1 \mathrm{mM})$ are $300 \mu \mathrm{M}$.

this study, the luciferase gene was used for the regulated gene, so that all riboswitch-fused mRNA constructed here can be directly used as biosensors. The luciferase expression of theo5 increased in response to theophylline concentrations (Fig. 6D). The detection limit of theo5 for theophylline was $3 \mu \mathrm{M}$, which is much lower than those of prokaryotic riboswitch-based in vitro biosensors using a theophylline-dependent aptazyme $(\sim 100 \mu \mathrm{M})$ (Ogawa and Maeda 2007, 2008b) and is comparable to that of a previous eukaryotic riboswitch-based in vitro biosensor using the same aptazyme (Ogawa 2009). It is also comparable to the detection limits of non-riboswitch biosensors for theophylline such as a modular aptameric sensor (Stojanovic and Kolpashchikov 2004) and a FRET-based biosensor (Endoh et al. 2009).

Other reporter proteins can also be used instead of luciferase. For examples, fluorescent proteins such as green fluorescent protein (GFP) are useful for constructing enzyme reaction-free biosensors. In fact, eGFP-based biosensors for theophylline and tetracycline were easily constructed by replacing the luciferase gene of theo5 and tc7, respectively, with the eGFP gene (theo5-eGFP and tc7-eGFP, respectively) (Fig. 8). They exhibited high switching efficiencies, which are comparable to that of luciferase-based biosensors, suggesting that the riboswitch part (5' UTR) functions independently from the gene sequence and could be fused to arbitrary genes to regulate their expression. Although there have been many inventive sensors using aptamers reported thus far (Navani and Li 2006; Willner and Zayats 2007; Cho et al. 2009), riboswitch-based sensors have advantages in being label-free (and detector-free in some cases) (Ogawa and Maeda 2007) due to their utilization of protein expression as the output.

The IRES-mediated pt-ON-riboswitches described herein also have possibilities for in vivo applications other than in vitro biosensors. As described in the introduction, ribozyme-free aptamerbased riboswitches have been reported to have a good compatibility on their functions between in vitro and in vivo, at least in a prokaryotic translation system (Muranaka et al. 2009), in contrast to ribozyme-related riboswitches (Ogawa and Maeda 2007, 2008a). Of course, there are large differences between prokaryotes and eukaryotes in many aspects including transcription and translation systems though the mechanism of prokaryotic RBS-mediated translation is more similar to that of eukaryotic IRES-mediated translation than that of eukaryotic $5^{\prime}$-cap-dependent translation. Therefore, some additional creative thinking (such as for effective mRNA expression and efficient IRES-mediated translation) might be required for in vivo applications of the present IRES-mediated pt-ON-riboswitches. However, the 5'-terminus-mediated translation of the IRES-based riboswitches that is thought to be most impeditive for their high switching efficiency should be inhibited by an appropriate $5^{\prime}$-SL sequence in vivo (Babendure et al. 2006) and additionally by the mimic genes in the PSIV IRES as well as upstream open reading frames that many eukaryotes have in some of their mRNA. As to the IRES, the PSIV IRES have been found to function in various eukaryotic translation systems (Sasaki and Nakashima 1999; Kanamori and Nakashima 2001; 
A
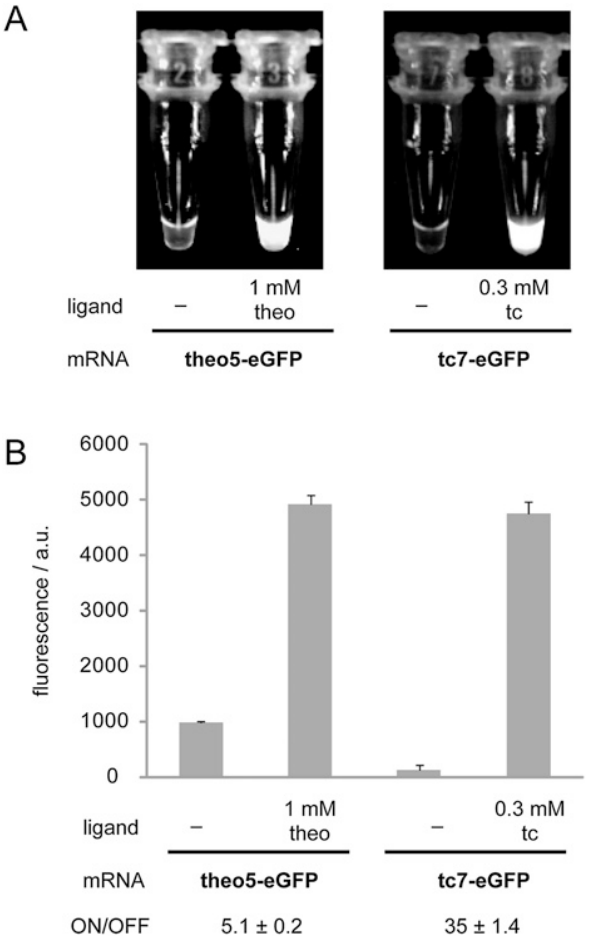

FIGURE 8. IRES-based riboswitches expressing eGFP. (A) Fluorescence images of eGFP translated from theo5-eGFP (left) or tc7-eGFP $($ right $)$ in the absence or presence of their corresponding ligands. $(B)$ The fluorescence intensities of eGFP shown in $A$. Each fluorescence value was calculated as the difference from an mRNA-free sample. Average ON/ OFF efficiencies with standard deviations are shown below the graph.

Shibuya et al. 2003). If the PSIV IRES does not function well in a certain eukaryote, other types of IRES whose activity is high in that organism and whose secondary structures are known (such as loops and/or pseudoknots required for efficient translation) could be used as the target of an aIRES to construct similar IRES-based riboswitches with the same procedure as for the PSIV IRES-based riboswitches. These riboswitches using the IRES originally present in various viruses are also useful for research regarding such viruses (Wang and White 2007). In vivo applications of the IRESbased riboswitches are now in progress.

In addition, the design strategy described here is available for constructing not only eukaryotic riboswitches but also prokaryotic ones by considering an aIRES as an anti-RBS. Many prokaryotic riboswitches have been obtained through selection methods. In most cases, however, it is difficult to use the selected riboswitch in response to a specific ligand for the design of other-ligand-dependent riboswitches due to problems derived from the fact that a part of the aptamer functions as the anti-RBS. On the other hand, the riboswitch constructed by the rational design strategy should completely separate the aptamer from the anti-RBS, so that the design of a certain optimized riboswitch could be easily applicable to that of various-ligand-dependent riboswitches as in the present study.

\section{MATERIALS AND METHODS}

\section{Preparation of DNA templates}

A primary DNA template for all PCRs was constructed by ligation between a PSIV IRES (Sasaki and Nakashima 1999) and the firefly luciferase gene, each of which was PCR-amplified from a pPSIV-IRES-NCP (artificially synthesized; GenScript) coding a part of the PSIV genome (5951-6204) and from a pE01Luc coding the F-Luc (Ogawa 2009), respectively. All DNA templates, including the T7 promoter sequence for transcription, were obtained with further PCRs by using the primary template as a template in the first PCR. DNA templates for riboswitches expressing eGFP were constructed by ligation between the $5^{\prime}$ UTR of luciferase-based riboswitches (theo5 or tc7) and the eGFP gene (artificially synthesized; GenScript), which has the same $3^{\prime}$ UTR as the luciferase-based riboswitches. All PCRs were performed by using PrimeSTAR Max DNA Polymerase (Takara Bio) with high fidelity.

\section{Preparation of mRNA templates}

Run-off transcription of the obtained DNA templates was performed with a MEGAscript T7 Kit (Applied Biosystems). The transcribed mRNA was purified with an RNeasy MinElute Cleanup Kit (QIAGEN) and quantified by the absorbance at $260 \mathrm{~nm}$. The purified mRNA, which had 251 nucleotides derived from the pE01Luc in the $3^{\prime}$ UTR, was used for translation without polyadenylation and $5^{\prime}$-capping (Ogawa 2009). It should be noted that mRNA without polyadenylation and/or $5^{\prime}$-capping is efficiently translated in wheat germ extract (Sawasaki et al. 2002; Kamura et al. 2005; Ogawa 2009). In addition, the ribosome loads on the $5^{\prime}$ terminus of mRNA even in the absence of the $5^{\prime}$ cap in wheat germ extract (Ogawa 2009).

\section{Prediction of secondary structures of mRNA}

The X-ray structure of PSIV IRES (Pfingsten et al. 2006) was referred for an IRES part. RNAstructure software (Mathews et al. 2004) was used for predicting a secondary structure of a $5^{\prime}$ part of mRNA.

\section{Translation in wheat germ extract}

Cell-free translation was carried out by using an WEPRO1240 Expression Kit (CellFree Sciences), as described previously (Ogawa 2009). A mixture $(10 \mu \mathrm{L})$ of the purified mRNA (3 pmol), various concentrations of ligands, WEPRO1240 (wheat germ extract, $2 \mu \mathrm{L}$ ), creatine kinase (final concentration: $40 \mathrm{ng} / \mu \mathrm{L}$ ), and SUB-AMIX (final concentration: $1 \times$ ), the latter three of which were attached to the kit, was incubated for $1 \mathrm{~h}$ at $26^{\circ} \mathrm{C}$.

\section{Luciferase assay}

An aliquot $(5 \mu \mathrm{L})$ of translation solution diluted 11-fold with water was mixed with Luciferase Assay Reagent containing luciferin (75 $\mu \mathrm{L}$; Promega). The chemiluminescence intensities were measured by using a Wallac ARVO MX (Perkin-Elmer) with a black 96-well plate. Chemiluminescence images were acquired using a Light-Capture (ATTO). 


\section{eGFP assay}

The fluorescence intensities of translation solution diluted fivefold with water were measured by using an excitation wavelength of $485 \mathrm{~nm}$ and an emission wavelength of $535 \mathrm{~nm}$ on a Wallac ARVO MX with a black 96-well plate. Fluorescence images were acquired using a Visirays-B (ATTO) and a Printgraph AE-6981 FXCP (ATTO) equipped with an appropriate filter (SCF515).

\section{ACKNOWLEDGMENTS}

This work was supported by "Special Coordination Funds for Promoting Science and Technology" from the Ministry of Education, Culture, Sports, Science and Technology, the Japanese Government.

Received August 24, 2010; accepted December 9, 2010.

\section{REFERENCES}

Babendure JR, Babendure JL, Ding JH, Tsien RY. 2006. Control of mammalian translation by mRNA structure near caps. RNA 12: 851-861.

Berens C, Thain A, Schroeder R. 2001. A tetracycline-binding RNA aptamer. Bioorg Med Chem 9: 2549-2556.

Breaker RR. 2002. Engineered allosteric ribozymes as biosensor components. Curr Opin Biotechnol 13: 31-39.

Burgstaller P, Famulok M. 1994. Isolation of RNA aptamers for biological cofactors by in vitro selection. Angew Chem Int Ed Engl 33: 1084-1087.

Cho EJ, Lee J-W, Ellington AD. 2009. Applications of aptamers as sensors. Annu Rev Anal Chem (Palo Alto Calif) 2: 241-264.

Desai SK, Gallivan JP. 2004. Genetic screens and selections for small molecules based on a synthetic riboswitch that activates protein translation. J Am Chem Soc 126: 13247-13254.

Endoh T, Shintani R, Mie M, Kobatake E, Ohtsuki T, Sisido M. 2009. Detection of bioactive small molecules by fluorescent resonance energy transfer (FRET) in RNA-protein conjugates. Bioconjug Chem 20: 2242-2246.

Grate D, Wilson C. 2001. Inducible regulation of the S. cerevisiae cell cycle mediated by an RNA aptamer-ligand complex. Bioorg Med Chem 9: 2565-2570.

Harvey I, Garneau P, Pelletier J. 2002. Inhibition of translation by RNA-small molecule interactions. RNA 8: 452-463.

Hellen CU, Sarnow P. 2001. Internal ribosome entry sites in eukaryotic mRNA molecules. Genes Dev 15: 1593-1612.

Holeman LA, Robinson SL, Szostak JW, Wilson C. 1998. Isolation and characterization of fluorophore-binding RNA aptamers. Fold Des 3: $423-431$.

Jenison RD, Gill SC, Pardi A, Polisky B. 1994. High-resolution molecular discrimination by RNA. Science 263: 1425-1429.

Kamura N, Sawasaki T, Kasahara Y, Takai K, Endo Y. 2005. Selection of $5^{\prime}$-untranslated sequences that enhance initiation of translation in a cell-free protein synthesis system from wheat embryos. Bioorg Med Chem Lett 15: 5402-5406.

Kanamori Y, Nakashima N. 2001. A tertiary structure model of the internal ribosome entry site (IRES) for methionine-independent initiation of translation. RNA 7: 266-274.

Kim DS, Gusti V, Pillai SG, Gaur RK. 2005. An artificial riboswitch for controlling pre-mRNA splicing. RNA 11: 1667-1677.

Kozak M. 1989. Context effects and inefficient initiation at non-AUG codons in eukaryotic cell-free translation systems. Mol Cell Biol 9: 5073-5080.

Madin K, Sawasaki T, Ogasawara T, Endo Y. 2000. A highly efficient and robust cell-free protein synthesis system prepared from wheat embryos: Plants apparently contain a suicide system directed at ribosomes. Proc Natl Acad Sci 97: 559-564.

Mathews DH, Disney MD, Childs JL, Schroeder SJ, Zuker M, Turner DH. 2004. Incorporating chemical modification constraints into a dynamic programming algorithm for prediction of RNA secondary structure. Proc Natl Acad Sci 101: 7287-7292.

Müller M, Weigand JE, Weichenrieder O, Suess B. 2006. Thermodynamic characterization of an engineered tetracycline-binding riboswitch. Nucleic Acids Res 34: 2607-2617.

Muranaka N, Sharma V, Nomura Y, Yokobayashi Y. 2009. Efficient design strategy for whole-cell and cell-free biosensors based on engineered riboswitches. Anal Lett 42: 108-122.

Navani NK, Li Y. 2006. Nucleic acid aptamers and enzymes as sensors. Curr Opin Chem Biol 10: 272-281.

Nomura Y, Yokobayashi Y. 2007. Reengineering a natural riboswitch by dual genetic selection. J Am Chem Soc 129: 13814-13815.

Ogawa A. 2009. Biofunction-assisted sensors based on a new method for converting aptazyme activity into reporter protein expression with high efficiency in wheat germ extract. ChemBioChem 10: 2465-2468.

Ogawa A, Maeda M. 2007. Aptazyme-based riboswitches as label-free and detector-free sensors for cofactors. Bioorg Med Chem Lett 17: 3156-3160.

Ogawa A, Maeda M. 2008a. An artificial aptazyme-based riboswitch and its cascading system in E. coli. ChemBioChem 9: 206-209.

Ogawa A, Maeda M. 2008b. A novel label-free biosensor using an aptazyme/suppressor-tRNA conjugate and an amber-mutated reporter gene. ChemBioChem 9: 2204-2208.

Pfingsten JS, Costantino DA, Kieft JS. 2006. Structural basis for ribosome recruitment and manipulation by a viral IRES RNA. Science 314: 1450-1454.

Roth A, Breaker RR. 2009. The structural and functional diversity of metabolite-binding riboswitches. Annu Rev Biochem 78: 305334.

Sasaki J, Nakashima N. 1999. Translation initiation at the CUU codon in mediated by the internal ribosome entry site of an insect picorna-like virus in vitro. J Virol 73: 1219-1226.

Sasaki J, Nakashima N. 2000. Methionine-independent initiation of translation in the capsid protein of an insect RNA virus. Proc Natl Acad Sci 97: 1512-1515.

Sawasaki T, Ogasawara T, Morishita R, Endo Y. 2002. A cell-free protein synthesis system for high-throughput proteomics. Proc Natl Acad Sci 99: 14652-14657.

Shibuya N, Nishiyama T, Kanamori Y, Saito H, Nakashima N. 2003. Conditional rather than absolute requirements of the capsid coding sequence for initiation of methionine-independent translation in Plautia stali intestine virus. J Virol 77: 12002-12010.

Shibuya N, Nishiyama T, Nakashima N. 2004. Cell-free synthesis of polypeptides lacking an amino-terminal methionine by using a dicistroviral intergenic internal ribosome entry site. J Biochem 136: 601-606.

Stojanovic MN, Kolpashchikov DM. 2004. Modular aptameric sensors. J Am Chem Soc 126: 9266-9270.

Suess B, Weigand JE. 2008. Engineered riboswitches. RNA Biol 5: 24-29.

Suess B, Hanson S, Berens C, Fink B, Schroeder R, Hillen W. 2003. Conditional gene expression by controlling translation with tetracycline-binding aptamers. Nucleic Acids Res 31: 18531858.

Suess B, Fink B, Berens C, Stentz R, Hillen W. 2004. A theophylline responsive riboswitch based on helix slipping controls gene expression in vivo. Nucleic Acids Res 32: 1610-1614.

Topp S, Gallivan JP. 2010. Emerging applications of riboswitches in chemical biology. ACS Chem Biol 5: 139-148.

Wang S, White KA. 2007. Riboswitching on RNA virus replication. Proc Natl Acad Sci 104: 10406-10411.

Weigand JE, Suess B. 2007. Tetracycline aptamer-controlled regulation of pre-mRNA splicing in yeast. Nucleic Acids Res 35: $4179-4185$. 
Weigand JE, Sanchez M, Gunnesch E-B, Zeiher S, Schroeder R, Suess B. 2008. Screening for engineered neomycin riboswitches that control translation initiation. RNA 14: 89-97.

Werstuck G, Green MR. 1998. Controlling gene expression in living cells through small molecule-RNA interactions. Science 282: 296-298.

Wieland M, Hartig JS. 2008. Improved aptazyme design and in vivo screening enable riboswitching in bacteria. Angew Chem Int Ed 47: 2604-2607.

Willner I, Zayats M. 2007. Electronic aptamer-based sensors. Angew Chem Int Ed 46: 6408-6418.
Wilson DS, Szostak JW. 1999. In vitro selection of functional nucleic acids. Annu Rev Biochem 68: 611-647.

Win MN, Smolke CD. 2007. A modular and extensible RNA-based gene-regulatory platform for engineering cellular function. Proc Natl Acad Sci 104: 14283-14288.

Win MN, Smolke CD. 2008. Higher-order cellular information processing with synthetic RNA devices. Science 322: 456-460.

Zimmermann GR, Jenison RD, Wick CL, Simorre J-P, Pardi A. 1997. Interlocking structural motifs mediate molecular discrimination by a theophylline-binding RNA. Nat Struct Biol 4: $644-649$. 

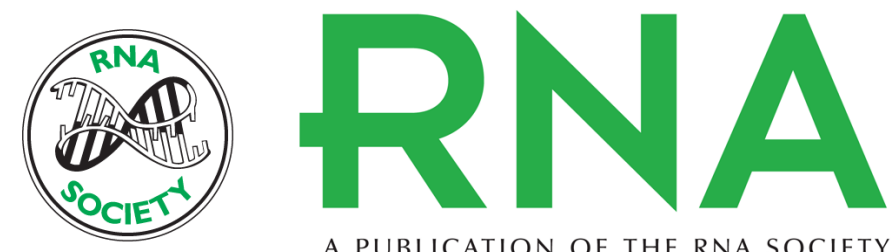

A PUBLICATION OF THE RNA SOCIETY

\section{Rational design of artificial riboswitches based on ligand-dependent modulation of internal ribosome entry in wheat germ extract and their applications as label-free biosensors}

Atsushi Ogawa

RNA 2011 17: 478-488 originally published online January 11, 2011

Access the most recent version at doi:10.1261/rna.2433111

References This article cites 48 articles, 19 of which can be accessed free at:

http://rnajournal.cshlp.org/content/17/3/478.full.html\#ref-list-1

License

Email Alerting Receive free email alerts when new articles cite this article - sign up in the box at the Service top right corner of the article or click here. 